OPEN ACCESS

Edited by: Valentina Morelli,

Istituto Auxologico Italiano, Italy

Reviewed by: Mateusz Maciejczyk, Medical University of Bialystok, Poland

*Correspondence: Xiaohong Xu

xuxiaoh@zju.edu.cn

Xiaoxiao Song

xsong103@zju.edu.cn

${ }^{\dagger}$ These authors have contributed equally to this work and share first authorship

${ }^{*}$ These authors have contributed equally to this work

Specialty section: This article was submitted to Adrenal Endocrinology, a section of the journal Frontiers in Endocrinology

Received: 18 October 2021 Accepted: 17 January 2022 Published: 09 February 2022

Citation:

Zhong S, Zhang T, He M, Yu H, LiU Z, Li Z, Song X and Xu X (2022) Recent Advances

in the Clinical Application of Adrenal Vein Sampling.

Front. Endocrinol. 13:797021. doi: 10.3389/fendo.2022.797021

\section{Recent Advances in the Clinical Application of Adrenal Vein Sampling}

\author{
Shan Zhong ${ }^{1+}$, Tianyue Zhang ${ }^{1 \dagger}$, Minzhi He${ }^{2}$, Hanxiao $\mathrm{Yu}^{3}$, Zhenjie $\mathrm{Liu}^{2}$, Zhongyi $\mathrm{Li}^{4}$, \\ Xiaoxiao Song ${ }^{1 *}$ and Xiaohong $\mathrm{Xu}^{1 * t}$ \\ ${ }^{1}$ Department of Endocrine and Metabolic Diseases, The Second Affiliated Hospital, Zhejiang University School of Medicine, \\ Hangzhou, China, ${ }^{2}$ Department of Vascular Surgery, The Second Affiliated Hospital School of Medicine, Zhejiang University \\ School of Medicine, Hangzhou, China, ${ }^{3}$ Clinical Research Center, The Second Affiliated Hospital, Zhejiang University School \\ of Medicine, Hangzhou, China, ${ }^{4}$ Department of Urinary Surgery, The Second Affiliated Hospital School of Medicine, Zhejiang \\ University School of Medicine, Hangzhou, China
}

We reviewed clinical research investigating the applications of adrenal vein sampling (AVS). AVS could be applied not only to primary aldosteronism (PA) but also to other endocrine diseases, such as adrenocorticotropic hormone (ACTH) independent Cushing syndrome (AICS) and hyperandrogenemia (HA). However, the AVS protocol requires improvements to increase its success rate. Using the computed tomography image fusion, coaxial guidewire technique, and fast intraprocedural cortisol testing (CCF) technique could improve the success rate of catheterization in AVS for PA. ACTH loading could be considered in medical centers with a low selectivity of AVS for PA but is not essential in those with mature AVS technology. The continuous infusion method should be recommended for ACTH stimulation in AVS for PA to reduce adverse events. AVS has not been routinely recommended before management decisions in AICS, but several studies verified that AVS was useful in finding out the source of excess cortisol, especially for distinguishing unilateral from bilateral disease. However, it is necessary to reassess the results of AVS in AICS with the use of reference hormones to fully normalize cortisol levels. In addition, it is essential to determine the optimal model that combines AVS results and mass size to guide the selection of surgical plans, including identifying the dominant gland and presenting the option of staged adrenalectomy, to minimize the impact of bilateral resection. For HA, AVS combined with ovarian intravenous sampling to locate excess androgens could be considered when imaging results are equivocal.

Keywords: adrenal vein sampling (AVS), primary aldosteronism (PA), Cushing syndrome (CS), hyperandrogenism (HA), adrenal

\section{INTRODUCTION}

Adrenal vein sampling (AVS) was first developed in the 1960s (1). The most important and extensive use of AVS was its use as the gold standard method for localizing the overproduction of aldosterone in primary aldosteronism (PA) (2). PA is the most common form of secondary hypertension. The sensitivity and specificity of AVS for detecting unilateral PA (UPA) were 95\% and $100 \%$, respectively $(2,3)$. Moreover, AVS could also be used for other endocrine disorders that are caused by excess secretion of cortisol or androgens from the adrenal gland. Adrenocorticotropic 
hormone (ACTH) independent Cushing syndrome (AICS) is mostly caused by cortisol-producing adrenocortical tumors. However, in some cases, it is difficult to accurately localize the responsible focuses, especially in the presence of bilateral adrenal masses. AVS might be ideal for distinguishing between the responsible focuses that are causing excess secretion of cortisol. Although hyperandrogenism (HA) is a common endocrine disorder among women, at times, imaging fails to detect the source of overproduction of androgens. Consequently, AVS could be an attractive alternative option for the etiological diagnosis of HA. Theoretically, AVS could be used for pheochromocytoma, although to the best of our knowledge, there are currently no articles on this. Despite AVS being safe and effective, the success rate has varied, ranging from 8 to $95 \%$, which has largely limited its wide application (4-6). Therefore, increasing the use of AVS requires improving the AVS protocol. Furthermore, the reference ranges are still controversial. In addition, the application of AVS in diseases other than PA is not yet known. Hence, in this review, we discussed in detail the latest improvements of AVS use in PA and briefly summarized the expanded application of AVS in AICS and HA, in order to help clinicians better understand the use of AVS in clinical practice. We searched all eligible original articles on PubMed with following keywords: (adrenal vein sampling [Title/ Abstract]) AND (primary hyperaldosteronism [Title/ Abstract]); (adrenal vein sampling [Title/Abstract]) AND Cushing syndrome [Title/Abstract]); (adrenal vein sampling [Title/Abstract]) AND hyperandrogenism [Title/Abstract]). There were no exclusion criteria in our review because the number of eligible studies was relatively small. The main results of representative studies are presented in Table $\mathbf{1 .}$

\section{PRIMARY ALDOSTERONISM}

Primary aldosteronism (PA) is the most common cause of secondary hypertension. The prevalence of PA ranges from 3.2 to $12.7 \%$ in primary practice and from 1 to $30 \%$ in referral centers. This mainly depends on the degree of hypertension in the population being examined. Excess aldosterone is a strong risk factor for heart and kidney damage, independent of sex, age, and blood pressure. The prevalence of PA among patients with recently detected hypertension in China was at least $4 \%$. It is critical to discriminate between the main subtypes to determine the correct therapeutic strategies, which is surgery for unilateral forms or medical therapy for bilateral forms (21-23). There has always been a degree of clinical difficulty in classifying the PA subtypes. Imaging often fails to visualize microadenomas and distinguish nonfunctioning incidentalomas from aldosterone-producing adenomas. The sensitivity and specificity of imaging were 78 and $75 \%$, respectively (2). Clinicians needed to comprehensively analyze the biochemical indicators, imaging manifestations, and AVS results. Despite AVS being the gold standard method and having high sensitivity and specificity for the diagnosis of different primary aldosteronism subtypes, it has varied success rates. Therefore, it is paramount to improve the success rate of AVS.
In 2016, the Endocrine Society Clinical Practice Guideline recommended that for PA, adrenal computed tomography (CT) should be performed before AVS, by an immobilized experienced AVS angiographer, with sufficient time for the operation and with simultaneous bilateral adrenal vein cannulations, while limiting the use of contrast during the procedure to help minimize the failure risk and postoperative complications (2). Many studies focused on increasing the success rate of AVS, including successful right adrenal vein catheterization, the role of ACTH loading during AVS, and the evaluation index of the AVS results, which we have described below.

\section{Correct Right Adrenal Vein Catheterization}

It is well known that AVS failure is often owing to unsuccessful catheterization of the right adrenal vein (the right adrenal vein is usually shorter than the left and typically enters the inferior vena cava (IVC) at an acute angle). A recent study at our center found that the Computed tomography image fusion, Coaxial guidewire technique, Fast intraprocedural cortisol testing (CCF) technique significantly improved technical success rates and reduced procedure time, radiation exposure, and contrast medium volume (7). Previously, the only access for AVS was via the femoral vein, but according to the study of J Xu et al., the forearm vein may provide a complementary or alternative approach to catheterization. The success rate of AVS of the right and left adrenal vein was 93.8 and $100 \%$, respectively (24). However, since only 48 patients were included in the study, the reliability of AVS access via the forearm vein remained unclear. Another viable method was using imaging to obtain an accurate visualization of the right adrenal vein, which was vital both before and during AVS. With the development of imaging technology, 3-dimensional reconstruction has frequently been applied in clinical settings. Using multi-detector CT (MDCT) with 3-dimensional reconstruction may reduce operation time and the quantity of contrast required and improve the success rate of catheterization. Previous studies have suggested that using Dyna CT, cone-beam CT during AVS could improve successful cannulation of the adrenal vein (25-27). Another study suggested that a 70-kilovoltage-peak $(\mathrm{kVp})$ contrastenhanced CT scan may provide better visualization and identification of the right adrenal vein (8). Thus, promoting the use of advanced CT techniques at medical centers may be necessary for visualizing the adrenal vein for accurate and successful cannulation.

\section{The Role of ACTH Loading During AVS}

At most medical centers, the medical staff did not possess the skills for simultaneous cannulation and as such, at about $40 \%$ of them the use of ACTH infusion during AVS is advocated for to overcome the limitations of non-synchronous catheterization $(28,29)$. A meta-analysis in 2018 showed that ACTH loading could significantly reduce the number of unsuccessful cannulations, without significantly increasing incorrect lateralization (9). However, a large retrospective study conducted by Tekada et al., in 2019 compared the two techniques (AVS with ACTH stimulation and AVS without 
TABLE 1 | Summary of main studies involving advances about application of AVS.

\begin{tabular}{llll}
\hline Authors, & Population & Results Lonclusions \\
year & &
\end{tabular}

Primary Aldosteronism (PA)

Correct right adrenal vein catheterization

Liu (7) $\quad 105$ Patients with PA (51 in the AVS-CCF group; 54 in the AVS

The technical success rate was higher for AVS-CCF than for AVS without CCF (98 vs. 83.3\% for bilateral adrenal veins, $P=0.016)$.

group)

Maruyama 90 Patients with PA (43

(8) in the 120-kVp group; 47 in the 70-kVp group)

In comparison with the $120-k V p$ group, the $70-k V p$ group had significantly superior conspicuity scores for the RAV $(P<0.001)$, higher RAV detection rates $(P=0.015-P=$ $0.033)$, and lower size-specific dose estimates $(P<0.001)$.

The role of ACTH loading during AVS

Laurent (9) 14 Studies comparing the 2 techniques (AVS with ACTH stimulation and AVS without ACTH stimulation in patient with PA)

Takeda 2197 Japanese patients (10) with PA from 28 centers

Hu (11) $\quad 174$ Patients with PA (80 receiving $\mathrm{ACTH}$ bolus; 94 receiving ACTH infusions)

The evaluation index of AVS results Li (12) 37 Patients with PA

Dekkers 86 Patients with PA (52

(13) in the cosyntropinstimulated group; 34 in the nonstimulated AVS group)
Wolley (14) 80 Patients with PA
AVS with ACTH stimulation significantly reduced the number of unsuccessful cannulations of both adrenal veins more than AVS without ACTH stimulation in patients with PA (OR: 0.26, 95\% Cl: 0.17, 0.40; P<0.00001).

ACTH loading during AVS improved the success rate from 67 to $89 \%$, while lateralization indices decreased from 62 to $28 \%$.

The $\mathrm{LI}$ and rate of complete biochemical remission (43/44, $97.7 \%$ vs 53/53, $100 \%, P=0.45$ ) did not significantly differ between the two groups. The bolus group reported more transient AEs such as palpitation (52.9\% vs 2.2\%) and abdominal discomfort ( $40.0 \%$ vs $2.2 \%$ ) than the infusion group.

$\mathrm{SI} \geq 3$ for androstenedione or DHEA provided optimal sensitivity and specificity in AVS. Given the much larger AV $\mathrm{PV}$ ratios and reduced variability compared to cortisol, the adrenal androgens are useful for assessing the selectivity of AVS without cosyntropin stimulation.

The adrenal to peripheral vein ratio of metanephrine was 6fold higher than that of cortisol (94.0 versus 15.5;

$\mathrm{P}<0.0001)$. ROC analysis indicated a plasma metanephrine SI cutoff of 12

The degree of contralateral suppression was independently and significantly correlated with postoperative SBP.
AVS with ACTH stimulation can significantly reduce the number of unsuccessful cannulations, without incorrect lateralization.

The use of ACTH during AVS was helpful for improving the success rate, but did not contribute to better outcomes.

Due to the similar effects on cannulation success and lateralization, but a lower rate of transient AEs in the infusion group, the continuous infusion method should be recommended for $\mathrm{ACTH}$ stimulation in AVS.

The adrenal androgens may be superior analytes in conditions with marked variability of cortisol levels or with cortisol and aldosterone.

Metanephrine provides a superior analyte compared with cortisol in assessing the selectivity of adrenal vein sampling during procedures without cosyntropin stimulation.

Contralateral suppression should be a factor in deciding whether to offer surgery for treatment of PA.

Combined ovarian and adrenal venous sampling is valuable in the localization of ACTH-independent ectopic CS. localize the source of autonomous cortisol hypersecretion in $\mathrm{ACTH}$-independent subclinical CS with bilateral adrenal masses.

AVS is of great significance for obtaining information on the functional state of BAAs before surgery. significantly reducing the number of adrenocortical tumors consecrating

AVS is a useful diagnostic tool that helps

The AVS-CCF procedures were performed more recently than the AVS without CCF procedures. The single-center, retrospective design, and use of 2 different CT scanners and different reconstruction techniques.

Variability in institutional protocols and shortage of expert interventional radiologists.

The limitation of the retrospective study.

The adrenal and peripheral venous blood before ACTH administration were not collected.

The sample size was small and the study did not compare the lateralization indices.

The sample size was small and the study did not compare the lateralization indices.

Patients without contralateral suppression were a relatively selected group.

The sample size. discovered by pelvic ultrasonography. Combined ovarian and adrenal venous sampling together with a cortiso assay were conducted. Results revealed a right-side ovarian origin.

Maghrabi a Patient with subclinical AVS results were consistent with bilateral autonomous cortisol hypersecretion without lateralization. A left adrenalectomy was performed. The patient improved clinically after the surgery.

Gu (17) a Patient with CS and

AVS results were consistent with bilateral autonomous cortisol hypersecretion without lateralization. A left adrenalectomy was performed, followed by resection of the right-side adrenal mass. 
TABLE 1 | Continued

\begin{tabular}{|c|c|c|c|c|}
\hline $\begin{array}{l}\text { Authors, } \\
\text { year }\end{array}$ & Population & Results & Conclusions & Limitations \\
\hline Raje (18) & $\begin{array}{l}6 \text { Patients with CS } \\
\text { ( } 3 \text { with bilateral adrenal } \\
\text { enlargement or nodules; } \\
3 \text { with unilateral nodules) }\end{array}$ & $\begin{array}{l}\text { AVS results aided management planning in five patients, } \\
\text { definitively changing treatment from surgery to medical } \\
\text { management in one patient. }\end{array}$ & $\begin{array}{l}\text { AVS offered useful information for } \\
\text { determining appropriate management of } \\
\text { adrenal CS. }\end{array}$ & The sample size. \\
\hline \multicolumn{5}{|c|}{ Hyperandrogenism (HA) } \\
\hline Tng (19) & $\begin{array}{l}3 \text { Studies including } \\
\text { women with HA who } \\
\text { underwent catherization }\end{array}$ & $\begin{array}{l}\text { The summary sensitivity of the dexamethasone } \\
\text { suppression test is } 100 \% \text { and that for selective venous } \\
\text { sampling is } 100 \% \text {. The summary specificity of the } \\
\text { dexamethasone suppression test is } 89.2 \% \text { and that for } \\
\text { selective venous sampling is } 100 \% \text {. }\end{array}$ & $\begin{array}{l}\text { There is limited evidence for the use of } \\
\text { selective venous sampling in identifying } \\
\text { virilizing tumors in postmenopausal } \\
\text { hyperandrogenism. }\end{array}$ & $\begin{array}{l}\text { Poor methodological } \\
\text { quality. }\end{array}$ \\
\hline $\begin{array}{l}\text { Kaltsas } \\
(20)\end{array}$ & $\begin{array}{l}38 \text { Patients who } \\
\text { underwent ovarian and } \\
\text { adrenal venous } \\
\text { catheterization and } \\
\text { sampling for } \\
\text { investigation of HA }\end{array}$ & $\begin{array}{l}\text { The overall catheterization success rate was: all four veins } \\
\text { in } 27 \% \text {, three veins in } 65 \% \text {, two veins in } 87 \% \text {. The success } \\
\text { rate for each individual vein was: right adrenal vein } 50 \% \text {, } \\
\text { right ovarian vein } 42 \% \text {, left adrenal vein } 87 \% \text { and left } \\
\text { ovarian vein } 73 \% \text {. }\end{array}$ & $\begin{array}{l}\text { Venous catheterization and sampling } \\
\text { should be considered only for patients in } \\
\text { whom uncertainty remains. }\end{array}$ & $\begin{array}{l}\text { The low successful } \\
\text { catherization rate. }\end{array}$ \\
\hline
\end{tabular}

AVS, adrenal vein sampling; PA, primary aldosteronism; CCF, computed tomography image fusion, coaxial guidewire technique, fast intraprocedural cortisol testing technique; RAV, right adrenal vein; CT, computerized tomography; $A C T H$, adrenocorticotropic hormone; $A E$, adverse event; $L I$, lateralization index; $C l$, confidence interval; SI, selective index; $R O C$, receiver operator characteristic; DHEA, dehydroepiandrosterone; AV, adrenal vein; PV, peripheral vein; SBP, systolic blood pressure; CS, Cushing syndrome; AIMAH, ACTH-independent macronodular adrenal hyperplasia; BAAs, bilateral adrenocortical adenomas; HA, hyperandrogenism.

ACTH stimulation among 2197 patients with PA from 28 centers in Japan and found that the use of ACTH loading during AVS increased the success rate from 67 to $89 \%$, while decreasing the lateralization rate from 62 to 28\% (10). Consequently, ACTH loading could be considered in medical centers with a low selectivity of AVS, but it is not essential in those with mature AVS technology. In addition, a recent study found that the continuous infusion method should be recommended for ACTH stimulation in AVS, due to the similar effects on cannulation success and lateralization, but a lower rate of transient adverse events among patients in the infusion group (11).

\section{The Evaluation Index of AVS Results}

The evaluation of AVS results comprised three indicators, including the selectivity index (SI), lateralization index (LI), and contralateral suppression index (CSI). In 2020, the European Society of Hypertension recommended that the SI of $>2$ for unstimulated and $>5$ for stimulated procedures be used to demonstrate correct cannulation of the adrenal veins. The LI of $>4$ for both unstimulated and stimulated procedures was considered unilateral PA. The CSI of $<1$ may indicate unilateral PA on the opposite side (22). However, criteria for AVS interpretation may vary between centers, owing to the large heterogeneity in AVS procedures and hormone measurements. Notably, although cortisol-based SI is currently a widely used indicator to evaluate the success of AVS, the secretion of cortisol varies $(28,30,31)$. Hence cortisol-based SI may not be the best indicator. With the higher adrenal vein (AV)/peripheral vein (PV) ratio of adrenal androgens, and more importantly, with the lower variability of adrenal androgens than cortisol, adrenal androgenbased SI may be more useful than cortisol-based SI for assessing the selectivity of non-ACTH stimulated AVS. It has also been demonstrated that adrenal androgen-based SI may be superior to cortisol-based SI in adrenal masses producing both aldosterone and cortisol (12). The study also found that the androgen-based SI of $\geq 3$ was an optimal cut-off point for assessing the selectivity of AVS. In comparison to catecholamines, there is relatively little increase in metanephrines in response to stress. Furthermore, compared with cortisol, metanephrines provided a superior analyte in assessing the selectivity of AVS, with a SI cut-off point of 12 (13). Although the LI was widely used to determine whether there was dominant secretion of aldosterone, CSI may be a good substitution for some cases where catheterization on the dominant side has been unsuccessful. A retrospective study conducted by Wolley et al., in 2015 showed that the CSI of $<1$ correlated with positive blood pressure and biochemical outcomes following surgery (14).

\section{ADRENOCORTICOTROPIC HORMONE INDEPENDENT CUSHING SYNDROME}

Endogenous Cushing syndrome (CS) is a rare and severe disease with an annual incidence of 0.2 to 5.0 per million and a prevalence of 39 to 79 per million (32). CS has a high mortality rate, with a standard mortality rate (SMR) of approximately 2.0 to 4.0; cardiovascular disease is the most common cause of CSrelated death (32). Adrenocorticotropic hormone independent CS (AICS) accounted for about $20 \%$ of CS cases, including unilateral adrenal adenoma or carcinoma, bilateral macronodular adrenal hyperplasia, bilateral micronodular adrenal hyperplasia, primary pigmented nodular adrenocortical disease, McCune-Albright syndrome, and bilateral adrenal adenomas or carcinomas. Among patients with AICS with bilateral adrenal masses, it is often difficult to determine the source of excess cortisol, which largely affected surgical planning. However, the source of excess cortisol in AICS could be localized using AVS. Multiple case 
reports have suggested that AVS may be used to successfully localize excess cortisol in patients with AICS (16, 33-37). In the case of a 40-year-old woman from China with a large pelvic mass, AVS also aided localization of ectopic AICS (15).

The interpretation of AVS results in AICS was controversial. Young et al., stated that epinephrine concentrations exceeding that of PV concentrations by more than $100 \mathrm{pg} / \mathrm{mL}$ indicates successful catheterization, an $\mathrm{AV} / \mathrm{PV}$ cortisol gradient of $>6.5$ indicates cortisol-secreting adrenal adenoma, and a high-side to low-side $\mathrm{AV}$ cortisol lateralization ratio of $\geq 2.3$ is consistent with autonomous cortisol secretion from predominantly one adrenal gland (38). A case report showed that a patient with subclinical CS and $\mathrm{ACTH}$-independent macronodular adrenal hyperplasia (AIMAH) underwent AVS and the result indicated no lateralization using criteria stated by Young et al. A left adrenalectomy was performed, and the patient improved clinically after the surgery (the left mass was larger than the right mass) (16). However, in another case with bilateral adrenocortical adenomas (BAAs) and bilateral autonomous cortisol secretion without laterality, the patient required left adrenalectomy followed by the resection of the right-side adrenal mass to gain clinical recovery (17). In both cases with similar AVS results, distinct management was necessary to cure CS, which posed challenges in the interpretation of AVS results, particularly in the question of how to choose the reference hormone to calculate the cortisol to reference hormone ratios from the right and left adrenal veins and finally calculate the lateralization index in non-synchronous catheterization approaches, due to the rapid fluctuation in adrenal hormones during the AVS procedure. In 2018, Wei Jie el at., reported that aldosterone concentrations could be used as reference hormones to calculate the lateralization index (36). However, many factors can interfere with aldosterone concentrations. In addition, aldosterone has a shorter half-life (20 min) than cortisol (60-70 $\mathrm{min}$ ) that may interfere with the interpretation of AVS findings. Therefore, aldosterone as a reference hormone still requires further validation (33). New criteria stated that a ratio of $>12$ for metanephrine was considered as correct cannulation. The LI of $\geq 2$ was interpreted as unilateral disease, using aldosterone, adrenaline, noradrenaline, and dehydroepiandrosterone sulfate as references $(18,39)$. Because universal criteria for the evaluation of laterality among patients with hypercortisolism in multi-centers have not yet been established, more multi-center studies are required. Moreover, in clinical practice in the presence of AIMAH, the excision of the larger mass is suggested in order to avoid bilateral adrenalectomy. Thus, it is essential to determine the optimal model that combines AVS results and mass size to guide the selection of surgical plans, to minimize the impact of bilateral resection.

\section{HYPERANDROGENISM}

Hyperandrogenism (HA) is a common endocrine disorder among women of a reproductive age, with a prevalence of approximately 5-10\% (40). Causes of HA included endogenous neoplasms, non-neoplastic androgen overproduction, and exogenous pharmacologic agents. Endogenous neoplasms included adrenocortical adenomas or carcinomas, SertoliLeydig cell tumors, hilus cell tumors, teratomas, pituitary adenomas, and others. Adrenal and ovary vein sampling could be considered to locate the source of excess androgen. However, there were only limited reports from studies with small sample size (19, 41-51). A study involving 38 patients with HA showed that the successful catheterization rate of the four veins (bilateral AVs and ovary veins (OVs)) was $27 \%$, and the failure rate of $\mathrm{OV}$ catheterization was higher than AV catheterization. OV/PV ratio of estradiol of $>2$ was considered as successful cannulation of the $\mathrm{OV} ; \mathrm{AV} / \mathrm{PV}$ ratio of cortisol of $>2$ was considered as successful cannulation of the AV; A ratio of testosterone $>2$ was considered as androgen overproduction (20). In this study, no complications were observed. However, the low success rate of catheterization limited the application of venous sampling in HA, thus it may be only considered when imaging results are equivocal.

\section{CONCLUSIONS}

Conclusions from our review are presented in a summary figure (Figure 1). AVS could be applied not only to PA but also to other endocrine diseases, such as AICS and HA. However, the AVS protocol requires improvements to increase its success rate. Using the CCF technique could improve the success rate of catheterization
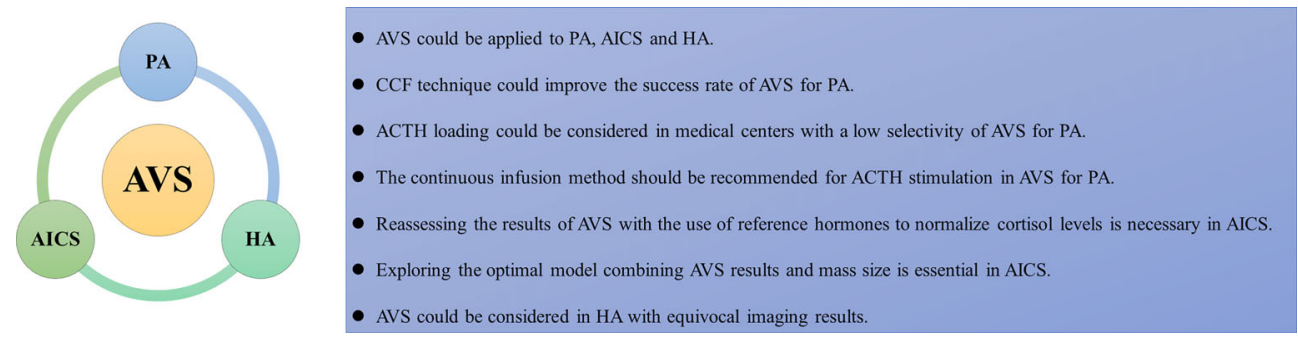

FIGURE 1 | The summarizing figure of the review. AVS, adrenal vein sampling; AICS, adrenocorticotropic hormone independent Cushing syndrome; HA, hyperandrogenism; CCF, computed tomography image fusion, coaxial guidewire technique, fast intraprocedural cortisol testing technique; ACTH, adrenocorticotropic hormone. 
in AVS for PA. ACTH loading could be considered in medical centers with a low selectivity of AVS for PA but is not essential in those with mature AVS technology. The continuous infusion method should be recommended for ACTH stimulation in AVS for PA to reduce adverse events. AVS hasn't been routinely recommended before management decisions in AICS, but several studies verified that AVS was useful to find out the source of excess cortisol, especially for distinguishing unilateral from bilateral disease. However, it is necessary to reassess the results of AVS in AICS with the use of reference hormones to fully normalize cortisol levels. In addition, it is essential to determine the optimal model that combines AVS results and mass size to guide the selection of surgical plans, including identifying the dominant gland and presenting the option of staged adrenalectomy to minimize the impact of bilateral resection. For HA, AVS combined with ovarian intravenous sampling to locate excess androgens could be considered when imaging results are equivocal. This study had some limitations. First, since this review only explored the PubMed database and only included articles written in English, some articles may have been missed. Second, most studies included had limited

\section{REFERENCES}

1. Lecky JW. Percutaneous Transjugular Approach to Adrenal Venography. Am J Roentgenol Radium Ther Nucl Med (1968) 104(2):380-5. doi: 10.2214/ ajr.104.2.380

2. Funder JW, Carey RM, Mantero F, Murad MH, Reincke M, Shibata H, et al. The Management of Primary Aldosteronism: Case Detection, Diagnosis, and Treatment: An Endocrine Society Clinical Practice Guideline. J Clin Endocrinol Metab (2016) 101(5):1889-916. doi: 10.1210/ jc.2015-4061

3. Young WF. Primary Aldosteronism: Renaissance of a Syndrome. Clin Endocrinol (Oxf) (2007) 66(5):607-18. doi: 10.1111/j.1365-2265.2007.02775.x

4. Young WF, Stanson AW, Thompson GB, Grant CS, Farley DR, van Heerden JA. Role for Adrenal Venous Sampling in Primary Aldosteronism. Surgery (2004) 136(6):1227-35. doi: 10.1016/j.surg.2004.06.051

5. Vonend O, Ockenfels N, Gao X, Allolio B, Lang K, Mai K, et al. Adrenal Venous Sampling: Evaluation of the German Conn's Registry. Hypertension (2011) 57(5):990-5. doi: 10.1161/hypertensionaha.110.168484

6. Monticone S, Satoh F, Dietz AS, Goupil R, Lang K, Pizzolo F, et al. Clinical Management and Outcomes of Adrenal Hemorrhage Following Adrenal Vein Sampling in Primary Aldosteronism. Hypertension (2016) 67(1):146-52. doi: 10.1161/hypertensionaha.115.06305

7. Liu Z, He M, Song X, Xu F, Zhang B, Chen B, et al. Computed Tomography Image Fusion, Coaxial Guidewire Technique, Fast Intraprocedural Cortisol Testing Technique Improves Success Rate and Decreases Radiation Exposure, Procedure Time, and Contrast Use for Adrenal Vein Sampling. J Hypertens (2021) 39(9):1918-25. doi: 10.1097/HJH.0000000000002852

8. Maruyama K, Sofue K, Horinouchi H, Okada T, Ueshima E, Gentsu T, et al. Improved Visualization and Identification of the Right Adrenal Vein in 70$\mathrm{kVp}$ Contrast-Enhanced Computed Tomography. J Comput Assist Tomogr (2020) 44(1):153-9. doi: 10.1097/RCT.0000000000000960

9. Laurent I, Astere M, Zheng F, Chen X, Yang J, Cheng Q, et al. Adrenal Venous Sampling With or Without Adrenocorticotropic Hormone Stimulation: A Meta-Analysis. J Clin Endocrinol Metab (2018) 104:1060-8. doi: 10.1210/ jc.2018-01324

10. Takeda Y, Umakoshi H, Takeda Y, Yoneda T, Kurihara I, Katabami T, et al. Impact of Adrenocorticotropic Hormone Stimulation During Adrenal Venous Sampling on Outcomes of Primary Aldosteronism. J Hypertens (2019) 37(5):1077-82. doi: 10.1097/hjh.0000000000001964

11. Hu J, Chen J, Cheng Q, Jing Y, Yang J, Du Z, et al. Comparison of Bolus and Continuous Infusion of Adrenocorticotropic Hormone During Adrenal Vein sample sizes. Hence, more research should be conducted to improve the understanding of the clinical application of AVS in endocrine diseases.

\section{AUTHOR CONTRIBUTIONS}

$\mathrm{XS}$ and XX came up with the idea. SZ and TZ wrote the main text. MH, HY, ZheL, and ZhoL collected information. All authors were involved in drafting the manuscript, and have read and approved the final version.

\section{FUNDING}

This work was supported by the National Natural Science Foundation of China (813000083), the Medicine and Health project founded by Zhejiang Province (2020380946, 2022502078), and the Science and Technology of Zhejiang Province Project (LGF21H070003).
Sampling. Front Endocrinol (Lausanne) (2021) 12:784706:784706. doi: 10.3389/ fendo.2021.784706

12. Li H, Zhang X, Shen S, Zhang Y, Zhang W, Feng W, et al. Adrenal Androgen Measurement for Assessing the Selectivity of Adrenal Venous Sampling in Primary Aldosteronism. Steroids (2018) 134:16-21. doi: 10.1016/ j.steroids.2018.04.002

13. Dekkers T, Deinum J, Schultzekool LJ, Blondin D, Vonend O, Hermus AR, et al. Plasma Metanephrine for Assessing the Selectivity of Adrenal Venous Sampling. Hypertension (2013) 62(6):1152-7. doi: 10.1161/HYPERTENSIONAHA. 113.01601

14. Wolley MJ, Gordon RD, Ahmed AH, Stowasser M. Does Contralateral Suppression at Adrenal Venous Sampling Predict Outcome Following Unilateral Adrenalectomy for Primary Aldosteronism? A Retrospective Study. J Clin Endocrinol Metab (2015) 100(4):1477-84. doi: 10.1210/jc.20143676

15. Chen S, Li R, Zhang X, Lu L, Li J, Pan H, et al. Combined Ovarian and Adrenal Venous Sampling in the Localization of Adrenocorticotropic HormoneIndependent Ectopic Cushing Syndrome. J Clin Endocrinol Metab (2018) 103(3):803-8. doi: 10.1210/jc.2017-01977

16. Maghrabi A, Yaqub A, Denning KL, Benhamed N, Faiz S, Saleem T. Challenges in the Diagnostic Work-Up and Management of Patients With Subclinical Cushing's Syndrome and Bilateral Adrenal Masses. Endocr Pract (2013) 19(3):515-21. doi: 10.4158/EP12277.RA

17. Gu YL, Gu WJ, Dou JT, Lv ZH, Li J, Zhang SC, et al. Bilateral Adrenocortical Adenomas Causing Adrenocorticotropic Hormone-Independent Cushing's Syndrome: A Case Report and Review of the Literature. World J Clin cases (2019) 7(8):961-71. doi: 10.12998/wjcc.v7.i8.961

18. Raje P, Broekhuis JM, Sacks BA, James BC. Diagnostic Impact of Adrenal Vein Sampling in Adrenal Cushing's Syndrome. J Surg Res (2021) 268:660-6. doi: $10.1016 /$ j.jss.2021.08.006

19. Tng EL, Tan JM. Dexamethasone Suppression Test Versus Selective Ovarian and Adrenal Vein Catheterization in Identifying Virilizing Tumors in Postmenopausal Hyperandrogenism - A Systematic Review and MetaAnalysis. Gynecol Endocrinol (2021) 37(7):600-8. doi: 10.1080/ 09513590.2021.1897099

20. Kaltsas GA, Mukherjee JJ, Kola B, Isidori AM, Hanson JA, Dacie JE, et al. Is Ovarian and Adrenal Venous Catheterization and Sampling Helpful in the Investigation of Hyperandrogenic Women? Clin Endocrinol (Oxf) (2003) 59 (1):34-43. doi: 10.1046/j.1365-2265.2003.01792.x

21. Mulatero P, Monticone S, Deinum J, Amar L, Prejbisz A, Zennaro MC, et al. Genetics, Prevalence, Screening and Confirmation of Primary Aldosteronism: 
A Position Statement and Consensus of the Working Group on Endocrine Hypertension of The European Society of Hypertension. J Hypertens (2020) 38 (10):1919-28. doi: 10.1097/HJH.0000000000002510

22. Mulatero P, Sechi LA, Williams TA, Lenders JWM, Reincke M, Satoh F, et al. Subtype Diagnosis, Treatment, Complications and Outcomes of Primary Aldosteronism and Future Direction of Research: A Position Statement and Consensus of the Working Group on Endocrine Hypertension of the European Society of Hypertension. J Hypertens (2020) 38(10):1929-36. doi: 10.1097/HJH.0000000000002520

23. Xu Z, Yang J, Hu J, Song Y, He W, Luo T, et al. Primary Aldosteronism in Patients in China With Recently Detected Hypertension. J Am Coll Cardiol (2020) 75(16):1913-22. doi: 10.1016/j.jacc.2020.02.052

24. Xu J, Sheng C, Li M, Shen W, Tang X, Zhu L, et al. A Feasibility Study on Percutaneous Forearm Vein Access for Adrenal Venous Sampling. J Hum Hypertens (2017) 31(1):76-8. doi: 10.1038/jhh.2016.41

25. Onozawa S, Murata S, Tajima H, Yamaguchi H, Mine T, Ishizaki A, et al. Evaluation of Right Adrenal Vein Cannulation by Computed Tomography Angiography in 140 Consecutive Patients Undergoing Adrenal Venous Sampling. Eur J Endocrinol (2014) 170(4):601-8. doi: 10.1530/EJE-13-0741

26. CC C, BC L, KL L, YC C, VC W, KH H. Non-Stimulated Adrenal Venous Sampling Using Dyna Computed Tomography in Patients With Primary Aldosteronism. Sci Rep (2016) 6:37143. doi: 10.1038/srep37143

27. O M, É A, MC D, FZ M, J D, H R, et al. Impact of Cone Beam - CT on Adrenal Vein Sampling in Primary Aldosteronism. Eur J Radiol (2020) 124:108792. doi: 10.1016/j.ejrad.2019.108792

28. Rossi GP, Barisa M, Allolio B, Auchus RJ, Amar L, Cohen D, et al. The Adrenal Vein Sampling International Study (AVIS) for Identifying the Major Subtypes of Primary Aldosteronism. J Clin Endocrinol Metab (2012) 97 (5):1606-14. doi: 10.1210/jc.2011-2830

29. Wolley MJ, Ahmed AH, Gordon RD, Stowasser M. Does ACTH Improve the Diagnostic Performance of Adrenal Vein Sampling for Subtyping Primary Aldosteronism? Clin Endocrinol (Oxf) (2016) 85(5):703-9. doi: 10.1111/ cen. 13110

30. Tanemoto M, Suzuki T, Abe M, Abe T, Ito S. Physiologic Variance of Corticotropin Affects Diagnosis in Adrenal Vein Sampling. Eur J Endocrinol (2009) 160(3):459-63. doi: 10.1530/EJE-08-0840

31. Seccia TM, Miotto D, Battistel M, Motta R, Barisa M, Maniero C, et al. A Stress Reaction Affects Assessment of Selectivity of Adrenal Venous Sampling and of Lateralization of Aldosterone Excess in Primary Aldosteronism. Eur J Endocrinol (2012) 166(5):869-75. doi: 10.1530/EJE-11-0972

32. Lacroix A, Feelders RA, Stratakis CA, Nieman LK. Cushing's Syndrome. Lancet (2015) 386(9996):913-27. doi: 10.1016/S0140-6736(14)61375-1

33. Martins RG, Agrawal R, Berney DM, Reznek R, Matson M, Grossman AB, et al. Differential Diagnosis of Adrenocorticotropic Hormone-Independent Cushing Syndrome: Role of Adrenal Venous Sampling. Endocr Pract (2012) 18(6):e153-157. doi: 10.4158/EP12136.CR

34. Ku EJ, Hong AR, Kim YA, Bae JH, Chang MS, Kim SW. Adrenocorticotropic Hormone-Independent Cushing Syndrome With Bilateral Cortisol-Secreting Adenomas. Endocrinol Metab (Seoul) (2013) 28(2):133-7. doi: 10.3803/ EnM.2013.28.2.133

35. Builes-Montano CE, Villa-Franco CA, Roman-Gonzalez A, Velez-Hoyos A, Echeverri-Isaza S. Adrenal Venous Sampling in a Patient With Adrenal Cushing Syndrome. Colomb Med (Cali) (2015) 46(2):84-7. doi: 10.25100/cm.v46i2.1938

36. Wei J, Li S, Liu Q, Zhu Y, Wu N, Tang Y, et al. ACTH-Independent Cushing's Syndrome With Bilateral Cortisol-Secreting Adrenal Adenomas: A Case Report and Review of Literatures. BMC Endocr Disord (2018) 18(1):22. doi: 10.1186/s12902-018-0250-6

37. Acharya R, Dhir M, Bandi R, Yip L, Challinor S. Outcomes of Adrenal Venous Sampling in Patients With Bilateral Adrenal Masses and ACTH-Independent Cushing's Syndrome. World J Surg (2019) 43(2):527-33. doi: 10.1007/s00268018-4788-2

38. Young WFJr., du Plessis H, Thompson GB, Grant CS, Farley DR, Richards $\mathrm{ML}$, et al. The Clinical Conundrum of Corticotropin-Independent Autonomous Cortisol Secretion in Patients With Bilateral Adrenal Masses. World J Surg (2008) 32(5):856-62. doi: 10.1007/s00268-007-9332-8
39. Papakokkinou E, Jakobsson H, Sakinis A, Muth A, Wangberg B, Ehn O, et al. Adrenal Venous Sampling in Patients With ACTH-Independent Hypercortisolism. Endocrine (2019) 66(2):338-48. doi: 10.1007/s12020-01902038-0

40. Azziz R, Sanchez LA, Knochenhauer ES, Moran C, Lazenby J, Stephens KC, et al. Androgen Excess in Women: Experience With Over 1000 Consecutive Patients. J Clin Endocrinol Metab (2004) 89(2):453-62. doi: 10.1210/jc.2003031122

41. Moltz L, Schwartz U, Sorensen R, Pickartz H, Hammerstein J. Ovarian and Adrenal Vein Steroids in Patients With Nonneoplastic Hyperandrogenism: Selective Catheterization Findings. Fertil Steril (1984) 42(1):69-75. doi: 10.1016/ s0015-0282(16)47960-4

42. Cserepes E, Szucs N, Patkos P, Csapo Z, Molnar F, Toth M, et al. Ovarian Steroid Cell Tumor and a Contralateral Ovarian Thecoma in a Postmenopausal Woman With Severe Hyperandrogenism. Gynecol Endocrinol (2002) 16(3):213-6. doi: 10.1080/gye.16.3.213.216

43. Ali FSM, Stanaway SERS, Zakhour HD, Spearing G, Bowen-Jones D. A Case of Hirsutism Due to Bilateral Diffuse Ovarian Leydig Cell Hyperplasia in a PostMenopausal Woman. Eur J Internal Med (2003) 14(7):432-3. doi: 10.1016/ s0953-6205(03)00141-9

44. Nishiyama S, Hirota Y, Udagawa Y, Kato R, Hayakawa N, Tukada K. Efficacy of Selective Venous Catheterization in Localizing a Small AndrogenProducing Tumor in Ovary. Med Sci Monit (2008) 14(2):CS9-12.

45. Bailey AP, Schutt AK, Carey RM, Angle JF, Modesitt SC. Hyperandrogenism of Ovarian Etiology: Utilizing Differential Venous Sampling for Diagnosis. Obstet Gynecol (2012) 120(2 Pt 2):476-9. doi: 10.1097/AOG.0b013e31825a711c

46. Dunne C, Havelock JC. Malignant Ovarian Sertoli-Leydig Cell Tumor Localized With Selective Ovarian Vein Sampling. J Minim Invasive Gynecol (2012) 19(6):789-93. doi: 10.1016/j.jmig.2012.08.006

47. Frysak Z, Karasek D, Hartmann I, Kucerova L. Secondary Hypertension and Hirsutism as a Clinical Manifestation of Tumor Duplicity. BioMed Pap Med Fac Univ Palacky Olomouc Czech Repub (2015) 159(1):163-5. doi: 10.5507/ bp. 2013.056

48. Tetsi Nomigni M, Ouzounian S, Benoit A, Vadrot J, Tissier F, Renouf S, et al. Steroidogenic Enzyme Profile in an Androgen-Secreting Adrenocortical Oncocytoma Associated With Hirsustism. Endocr Connect (2015) 4(2):11727. doi: 10.1530/EC-15-0014

49. Hickman LC, Goodman L, Falcone T. Value of Selective Venous Catheterization in the Diagnosis of Hyperandrogenism. Fertil Steril (2017) 108(6):1085. doi: 10.1016/j.fertnstert.2017.08.037

50. Boehnisch M, Lindner U, Salameh T, Gebbert A, Kaltofen L, Krah M, et al. Multilocular Pure Leydig Cell Tumor of Ovary, Fallopian Tube, and Extraovarian Soft Tissue. AACE Clin Case Rep (2019) 5(1):e16-21. doi: 10.4158/ACCR-2018-0240

51. LeVee A, Suppogu N, Walsh C, Sacks W, Simon J, Shufelt C. The Masquerading, Masculinizing Tumor: A Case Report and Review of the Literature. J Womens Health (Larchmt) (2021) 30(7):1047-51. doi: 10.1089/ jwh.2020.8548

Conflict of Interest: The authors declare that the research was conducted in the absence of any commercial or financial relationships that could be construed as a potential conflict of interest.

Publisher's Note: All claims expressed in this article are solely those of the authors and do not necessarily represent those of their affiliated organizations, or those of the publisher, the editors and the reviewers. Any product that may be evaluated in this article, or claim that may be made by its manufacturer, is not guaranteed or endorsed by the publisher.

Copyright (c) 2022 Zhong, Zhang, He, Yu, Liu, Li, Song and Xu. This is an open-access article distributed under the terms of the Creative Commons Attribution License (CC BY). The use, distribution or reproduction in other forums is permitted, provided the original author(s) and the copyright owner(s) are credited and that the original publication in this journal is cited, in accordance with accepted academic practice. No use, distribution or reproduction is permitted which does not comply with these terms. 